\title{
Gram-Scale Synthesis of the N-Phenyl Phenothiazine Photocatalyst by Benzyne Addition
}

\author{
Erin N. Welsh ${ }^{\mathrm{a}}$, Katherine N. Robertson ${ }^{\mathrm{b}}$, Alexander W. H. Speed ${ }^{\mathrm{a} *}$ \\ ${ }^{a}$ Department of Chemistry, Dalhousie University, Halifax, Nova Scotia, Canada B3H 4R2 \\ ${ }^{b}$ Department of Chemistry, Saint Mary's University, Halifax, Nova Scotia, Canada B3H 3C3
}

\begin{abstract}
N-phenyl phenothiazine is one of the most reducing photoredox catalysts. Its synthesis commonly requires transition metal catalyzed cross-coupling reactions. Here we show the syntheses of four aryl phenothiazines via a benzyne route, including a multi-gram scale synthesis of N-phenyl phenothiazine. While yields are modest, the simplicity, low cost, and lack of requirement for cross-coupling catalyst in this synthesis will be attractive to users of this photocatalyst.
\end{abstract}

Introduction: N-aryl phenothiazines have recently emerged as strongly reducing metal-free photoredox catalysts. ${ }^{1}$ While highly active precious metal containing polypyridyl catalysts are commonly used in photoredox catalysis, metal-free catalysts are attractive for reasons of sustainability. ${ }^{2,3}$ In addition, phenothiazines are some of the strongest-reducing photoredox catalysts yet reported. ${ }^{4}$ As an example, N-phenyl phenothiazine is the only photoredox catalysts that has been shown to add $\mathrm{SF}_{5}$ to alkenes via reduction of $\mathrm{SF}_{6}{ }^{5}$ Aryl phenothazines have additionally been used as photocatalysts for atom-transfer, ${ }^{6}$ atom-transfer radical polymerization reactions, ${ }^{7}$ lignin reduction, ${ }^{8}$ carbofunctionalization, ${ }^{9}$ and olefin-alcohol addition. ${ }^{10}$

Phenothiazine radical cations have also been shown to be powerful super-oxidants, especially in their excited states. ${ }^{11}$
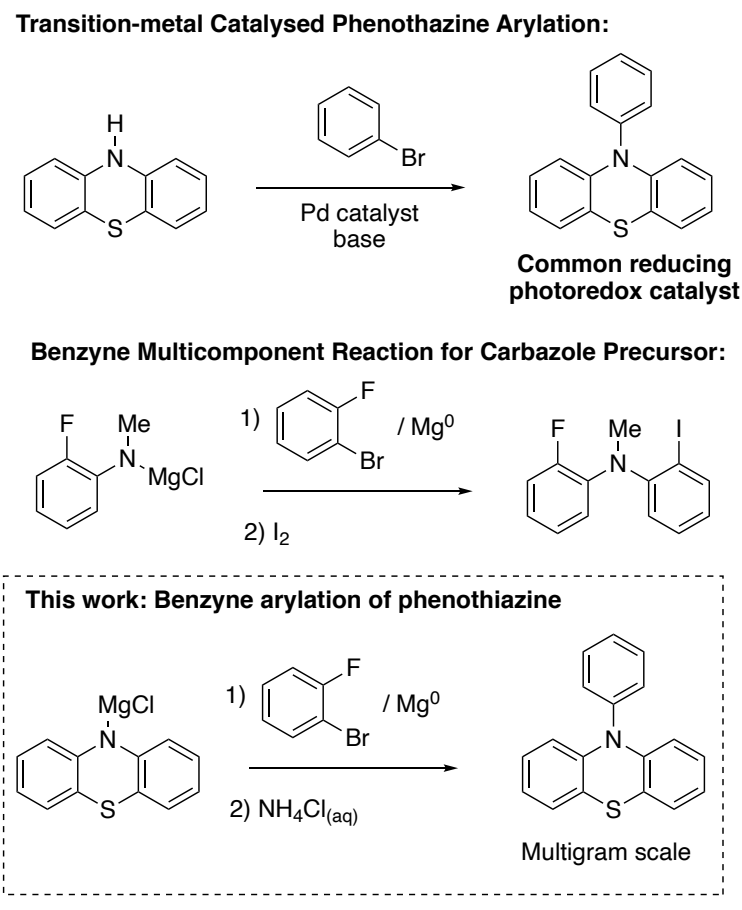

Scheme 1. N-Arylation of Relevance to Phenothiazine Synthesis 
While being metal-free themselves, aryl phenothiazines commonly involve precious metal catalysts in their synthesis, namely via a Buchwald-Hartwig amination for the $\mathrm{N}$-arylation. ${ }^{12,13}$ Palladium is expensive, and subject to price volatility, and expensive or sensitive ligands are required. In addition, transition metal trace contamination has been implicated as the true catalytic species in some allegedly metal-free catalytic systems. ${ }^{14}$ While this phenomenon has not yet been reported as an issue for organic photoredox catalysis, the rise of metallaphotoredox chemistry does make precious metal contamination a possibility that should be considered with caution. $^{15}$

Our group recently reported an expedited construction of dibenzothiophenes using a multicomponent reaction involving thiophenol addition to benzynes, ${ }^{16}$ which greatly simplified prior work that involved cross-coupling. ${ }^{17}$ Our work included one example relevant to carbazole synthesis involving the preparation of a diarylamine via addition of an anilide anion to benzyne. We were curious if this carbon-nitrogen bond formation could be expanded to include the formation of aryl phenothiazines, leading to a transition metal-free synthesis of N-phenyl phenothiazine. ${ }^{18} \mathrm{We}$ entered the project anticipating this would be a more challenging reaction, hypothesizing that the phenothiazide anion would be less nucleophilic than the anilides. This is due to the greater steric demand and the less-basic nitrogen bearing two rather than one aryl groups.
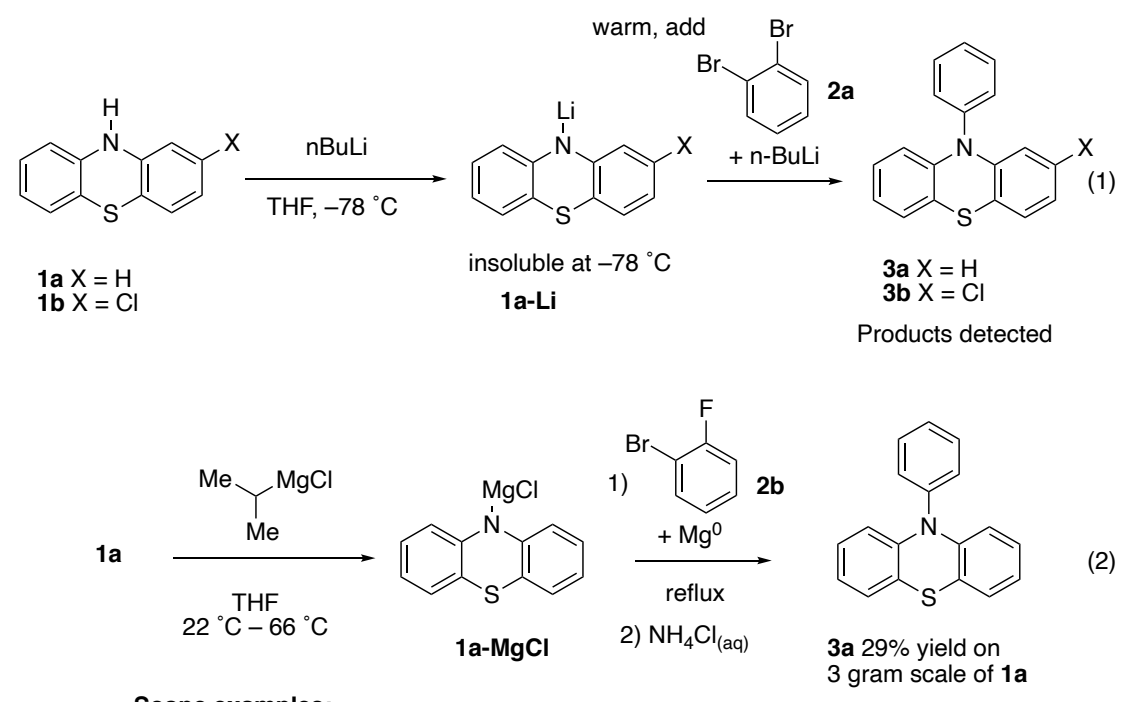

Scope examples:
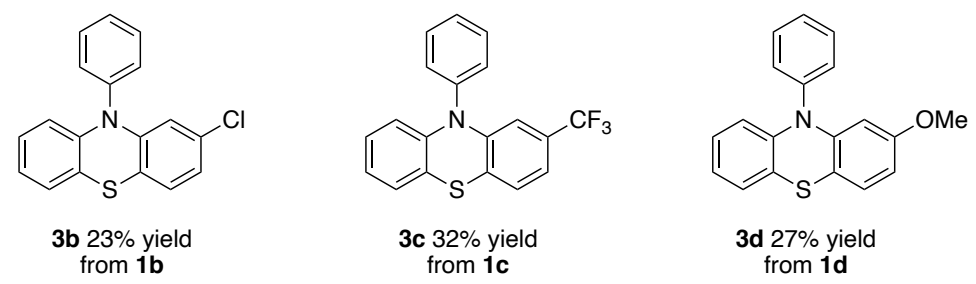

Scheme 2. Development of a benzyne arylation approach to aryl phenothiazines 
Results and Discussion: Initial attempts to deprotonate phenothiazine 1a or 2chlorophenothiazine $\mathbf{1 b}$ with n-butyllithium resulted in formation of precipitates at $-78{ }^{\circ} \mathrm{C}$ (Equation 1, Scheme 2). With most of the phenothiazide anion 1a-Li not in solution, rapid addition to the benzyne formed by addition of n-butyllithium to 1,2-dibromobenzene 2a would not occur. Warming the reaction mixture until the lithiated phenothiazine dissolved by removing the flask from the dry-ice bath, then addition of $\mathbf{2 a}$ and n-BuLi did result in the formation of aryl phenothiazines 3a and $\mathbf{3 b}$, detected by NMR spectroscopy of the crude reaction mixtures. Despite this observed product formation, we decided attempts to further optimize and scale up this procedure in batch would be unwise, due to the potential for a runaway exotherm during the benzyne formation from lithiated precursors and difficulty of balancing the appropriate temperature with the equipment available to us. ${ }^{1920}$

We then investigated an organomagnesium route to the benzyne addition at high temperature. Here the rate of benzyne formation is controlled by addition of the benzyne precursor, rather than rate of dissipation of heat from the reaction mixture. The magnesiated phenothiazine $1 \mathrm{a}-\mathrm{MgBr}$ is soluble in THF, and benzyne is formed by addition of the benzyne precursor 1-bromo-2-

fluorobenzene $\mathbf{2 b}$ to magnesium turnings already present in the reaction mixture. In our previous work, we found $\mathbf{2 b}$ to be a higher-yielding benzyne precursor than $\mathbf{2 a}$ under magnesiation conditions. Magnesiation of phenothiazine 1a with isopropylmagnesium chloride in the presence of magnesium turnings followed by slow addition of $\mathbf{2 b}$ led to formation of the desired product in $29 \%$ yield after purification on a 3 gram scale of 1a. Despite the low yield 1.2 grams of $\mathbf{2 a}$ was prepared on the first attempt (Scheme 2, Equation 2). Some limited substitution was tolerated, shown in Scheme 2. The 2-chlorophenothazine $\mathbf{1 b}$ could be used in place of 1a, with the corresponding arylated phenothiazine $\mathbf{3 b}$ being formed in $23 \%$ yield. ${ }^{21}$ No dechlorination to form 1a was observed in the isolated product. An electron-withdrawing $\mathrm{CF}_{3}$ group was tolerated, with 2-trifluoromethylphenothiazine 1c giving a 32\% yield of arylated phenothiazine $\mathbf{3 c}$. An electron donating methoxy group was also tolerated, with phenothiazine 3d arising from 2methoxy phenothiazine 1d in $27 \%$ yield. 


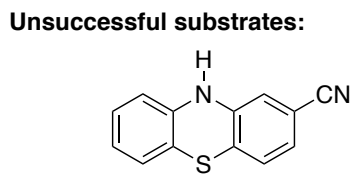

1e

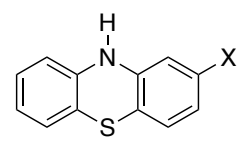

1f $X=S M e$

$\lg X=$ SEt

Dealkylation of 1f:<smiles>Cc1ccc2c(c1)Nc1ccccc1S2</smiles>

$1 f$

$22{ }^{\circ} \mathrm{C}-66{ }^{\circ} \mathrm{C} \quad \mathbf{1 f}-\mathrm{MgBr}$

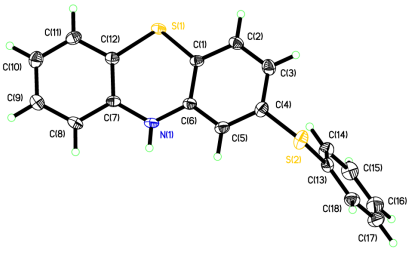

Structure of 4

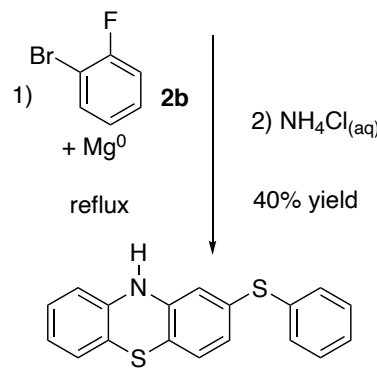

4
(1)

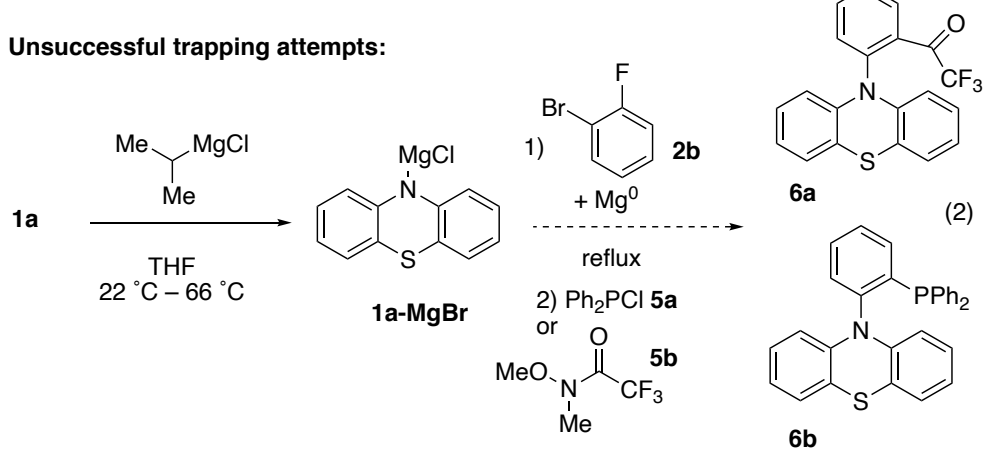

\section{Scheme 3. Limitations in Scope}

A 2-cyanophenothiazine 1e (Scheme 3) proved unreactive, potentially due to the attenuated nucleophilicity of the phenothiazide anion. Phenothiazine thioethers $\mathbf{1 f}$ and $\mathbf{1 g}$ were reactive, but gave an unexpected product. Dealkylation of these substates, and arylation of the thio-group to form 4 was observed, based on signals observed in NMR spectroscopy. Furthermore, crystals grew from ethyl acetate and hexanes fractions during chromatographic purification of 4 . Two polymorphs were observed by X-ray-crystallography which confirmed the identity of the product. In a specific example of this dealkylation, methyl thioether $1 \mathbf{f}$ was transformed to a phenylthioether 4 in 40\% yield, while the nitrogen remained unarylated (Equation 1, Scheme 3). Addition of the benzyne to the thioether, followed by reductive removal of the methyl group presumably occurred however no other reaction products containing the methyl group could be identified. ${ }^{22}$ Notably the methyl group was not transferred to either the nitrogen or introduced phenyl ring, as evidenced by both NMR and X-ray analysis. Reactions of aryl methyl thioethers, ${ }^{23}$ and other thioethers with benzyne to give either ylides or phenyl thioethers has been 
reported previously. ${ }^{24}$ Ethyl thioether $\mathbf{1 g}$ gave the same product, showing the reaction was not limited to the small methyl group. This unexpected outcome did alert us to another undesirable reaction mode that we had not initially anticipated, but did not observe: in none of the reactions were products arising from benzyne attack on endocyclic sulfur noted. Ylides derived from functionalization of the sulfur of aryl phenothiazines have previously been reported. ${ }^{25}$

Further attempted transformations by quenching with an electrophile other than a proton revealed this could not readily be turned into a three-component coupling. Attempting to quench the presumed organomagnesium intermediate formed after addition of the phenothiazide to the benzyne with either diphenylchlorophosphine $\mathbf{5 a}$ or Weinreb amide $\mathbf{5 b}$ did not result in productive formation of compounds $\mathbf{6 a}$ or $\mathbf{6 b}$ (Equation 2, Scheme 3). This is potentially a consequence of the high steric demand at the ortho position of the phenyl phenothiazine.

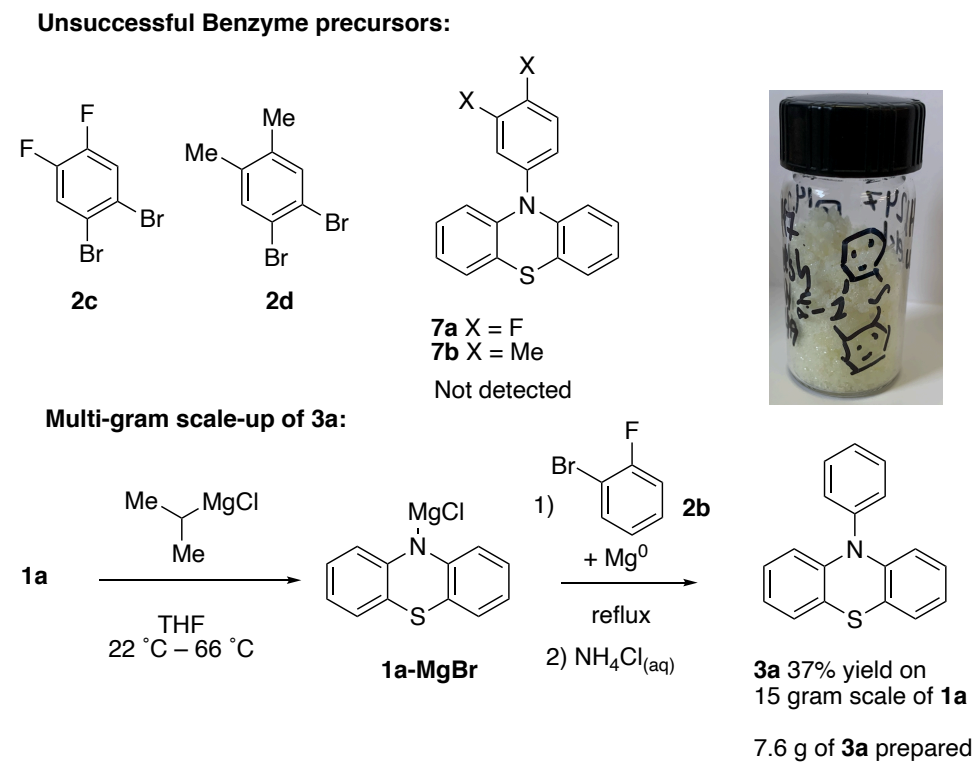

Scheme 4. Alternate benzyne precursors and scale-up

Investigation of dibromobenzene benzyne precursors was also not fruitful. Neither difluorodibromobenzene $\mathbf{2}$ or dibromoxylene $\mathbf{2 d}$ gave carbon-nitrogen bond formation under the same conditions (products 7a and 7b in shown in Scheme 4 were not observed). Compound 2d had been a successful substrate in our earlier multi-component synthesis of dibenzothiophenes via a benzyne route using organolithiums. ${ }^{16}$ In these reactions, consumption of the magnesium was notably slower than with $\mathbf{2 b}$, with overnight reflux being required. Modified entrainment conditions where dibromoethane was mixed with either $\mathbf{2 c}$ or $\mathbf{2 d}$ in THF solution resulted in more rapid magnesium consumption, but still did not lead to products $\mathbf{7 a}$ and $\mathbf{7 b}$. In both cases, consumption of the magnesium was observed, but unreacted phenothiazine was recovered from the reaction mixture.

In a final reaction, we scaled up the synthesis of 3a. A reaction on a 15 gram scale of starting material 1a was conducted. Here the yield of purified product 3a (shown in Scheme 4) increased to $37 \%$, providing 7.6 grams of material. 
While the yield of the reaction from starting material 1a is not comparable with some previously reported Buchwald-Hartwig couplings, this synthesis uses economical reagents, and no precious metal catalysts, so is cost-effective given the very inexpensive starting materials. It is likely that the increased cost of the more expensive substituted phenothiazines 1c and 1d would justify the use of higher-yielding protocols with more expensive catalysts to conduct the arylation. Such catalytic protocols would also likely be successful with substrates 1e-1f, which did not successfully react with benzyne. However, to the best of our knowledge this is the largest scale preparation of $\mathbf{3} \mathbf{a}$ that has been reported in the literature.

Conclusion: In conclusion, we report a synthesis of four aryl phenothiazine derivatives, prepared without using transition metal catalyzed cross-coupling While we have shown the scope of this approach is relatively limited, it performs admirably in the synthesis of phenyl phenothiazine 3a. Compound 3a is the most commonly used phenothiazine photoredox catalyst, and was prepared on a 7 gram scale in this work from inexpensive starting materials. This route will be of utility to other chemists using this photocatalyst.

\section{Corresponding Author}

*E-mail: aspeed@dal.ca

\section{Funding Sources}

This work was supported by NSERC of Canada through a Discovery Grant (2017-04297) and the CREATE Training Program in BioActives (510963). Labrador's Post-Secondary Student Support Program and the Nova Scotia Graduate Scholarship are thanked for E.N.W.'s graduate funding.

\section{ACKNOWLEDGMENT}

Dr. Michael Lumsden and Mr. Xiao Feng (Dalhousie University) are thanked for assistance with NMR spectroscopy and mass spectrometry, respectively.

\section{References:}

\footnotetext{
${ }^{1}$ Discekici, E. H.; Treat, N. J.; Poelma, S. O.; Mattson, K. M.; Hudson, Z. M.; Luo, Y.; Hawker, C. J.; Read de Alaniz, J. Chem. Commun. 2015, 51, 11705-11708.

${ }^{2}$ Romero, N. A.; Nicewicz, D. A. Chem. Rev. 2016, 116, 10075-10166.

${ }^{3}$ Du, Y.; Pearson, R. M.; Lim, C.-H.; Sartor, S. M.; Ryan, M. D.; Yang, H.; Damrauer, N. H.; Miyake, G. M. Chem. Eur. J. 2017, 23, 10962-10968.

${ }^{4}$ Speck, F.; Rombach, D.; Wagenknecht, H.-A. Beilstein J. Org. Chem. 2019, 15 52-59.

${ }^{5}$ A) Rombach, D.; Wagenknecht, H.-A. Chem. Cat. Chem. 2018, 10, 2955-2961. B) Rombach, D.; Wagenknecht, H.-A. Angew. Chem., Int. Ed. 2020, 59, 300-303.

${ }^{6}$ A) Steiner, A.; Williams, J. D.; Rincón, J. A.; de Frutos, O.; Mateos, C.; Kappe, C. O. Eur. J. Org. Chem. 2019, 5807-5811. B) Garrido-Castro, A.; Salaverri, N.; Maestro, M. C.; Alemán, J. Org. Lett. 2019, 21, 5295-5300. C) Shibutani, S.; Kodo, T.; Takeda, M.; Nagao, K.; Tokunaga, N.; Sasaki, Y.; Ohmiya, H. J. Am. Chem. Soc. 2020, 142, 1211-1216.

${ }^{7}$ A) Pan, X.; Fang, C.; Fantin, M.; Malhotra, N.; Young So, W.; Peteanu, L. A.; Isse, A. A.; Gennaro, A.; Liu, P.; Matyjaszewski, K. J. Am. Chem. Soc. 2016, 138, 2411-2425. B) Dadashi-Silab, S.; Pan, X.; Matyjaszewski, K. Chem. Eur. J. 2017, 23, 5972-5977.

${ }^{8}$ A)Li, H.; Bunrit, A.; Lu, J.; Gao, J.; Luo, N.; Liu, H.; Wang, F. ACS Catal. 2019, 9, 8843-8851. B)Yang, C.; Kärkäs, M. D.; Magallanes, G.; Chan, K.; Stephenson, C. R. J. Org. Lett. 2020, 22, 8082-8085.

${ }^{9}$ Shibutani, S.; Nagao, K.; Ohmiya, H. Org. Lett. 2021, 23, 1798-1803.

${ }^{10}$ Seyfert, F.; Wagenknecht, H. A. Synlett, 2021, 32, 582-586.
} 
${ }^{11}$ A) Christensen, J. A.; Phelan, B. T.; Chaudhuri, S.; Acharya, A.; Batista, V. S.; Wasielewski, M. R. J. Am. Chem. Soc. 2018, 140, 5290-5299. B) Targos, K.; Williams, O. P.; Wickens, Z. K. J. Am. Chem. Soc. 2021, 143, 41254132.

${ }^{12}$ A) Borowicz, P.; Herbich, J.; Kapturkiewicz, A.; Anulewicz-Ostrowska, R.; Nowacki, J.; Grampp, G. Phys. Chem. Chem. Phys. 2000, 2, 4275-4280. B) Mayer, L.; May, L.; Müller, T. J. J. Org. Chem. Front. 2020, 7, $1206-$ 1217.

${ }^{13}$ Sartor, S. M.; Chrisman, C. H.; Pearson, R. M.; Miyake, G. M.; Damrauer, N. H. J. Phys. Chem. A. 2020, 124, 817-823.

${ }^{14}$ A) Arvela, R. K.; Leadbeater, N. E.; Sangi, M. S.; Williams, V. A.; Granados, P.; Singer, R. D. J. Org. Chem. 2005, 70, 161-168. B) Avanthay, M.; Bedford, R.; Begg, C.; Böse, D.; Clayden, J.; Davis, S.; Eloi, J.-C.; Goryunov, G. P.; Hartung, I. V.; Heeley, J.; Khaikin, K. A.; Kitching, M.; Krieger, J.; Kulyabin, P. S.; Lennox, A.; NollaSaltiel, R.; Pridmore, N. E.; Rowsell, B. J. S.; Sparkes, H. A.; Uborsky, D. V.; Voskoboynikov, A. Z.; Walsh, M.; Wilkinson, H. J. Amine-Catalysed Suzuki-Miyaura-Type Coupling? the Identification and Isolation of the Palladium Culprits. ChemRxiv 2021. DOI: 10.26434/chemrxiv.14237288.v1

${ }^{15}$ Gualandi, A.; Anselmi, M.; Calogero, F.; Potenti, S.; Bassan, E.; Ceroni, P.; Cozzi, P. G. Org. Biomol. Chem. 2021, 19, 3527-3550.

${ }^{16}$ Welsh, E. N.; Robertson, K. N.; Speed, A. W. H. Org. Biomol. Chem. 2021, 19, 2000-2007.

${ }^{17}$ Sanz, R.; Fernández, Y.; Castroviejo, M. P.; Pérez, A.; Fañanás, F. J. J. Org. Chem. 2006, 71, 6291-6294.

${ }^{18}$ Conceptually distinct route to phenothiazines that involves a benzyne multicomponent reaction to establish the 1,2 S,N substutition pattern, followed by palladium-catalyzed C-N cyclization have been reported: A) Matsuzawa, T.; Uchida, K.; Yoshida, S.; Hosoya T. Chem. Lett. 2018, 47, 825-828. B) Matsuzawa, T.; Hosoya, T.; Yoshida, S. Org. Lett. 2021, 23, 2347-2352.

${ }^{19}$ Garcia-López, J.-A.; Greaney, M. F. Chem. Soc. Rev. 2016, 45, 6766-6798.

${ }^{20}$ It is likely the lithium route could be made viable by either flow chemistry, or a temperature-controlled chiller, neither of which we have ready access to.

${ }^{21}$ Xiang, S.; Huang, Z.; Sun, S.; Lv, X.; Fan, L.; Ye, S.; Chen, H.; Guo, R.; Wang, L. J. Mater. Chem. C. 2018, 6, $11436-11443$.

${ }^{22}$ Hori, M.; Kataoka, T.; Shimizu, H.; Ueda, N. Tet. Lett. 1981, 22, 3071-3074.

${ }^{23}$ Nakayama, J.; Fujita, T.; Hoshino, M. Chem. Lett. 1982, 1777-1780.

${ }^{24}$ A Blackburn, G. M.; Ollis, W. D. Chem. Commun. 1968, 1261-1262. B) Xu, H.-D.; Cai, M.-Q.; He, W.-J.; Hu, W.-H.; Shen, M.-H. RSC Adv. 2014, 4, 7623-7626.

${ }^{25}$ Liu, C.; Lin, Y.; Cai, C.; Yuan, C.; Fang, Z.; Guo, K. Green Chem. 2021, 23, 2956-2961. 